\title{
Size Modification of Nanographite System of Activated Carbon Fibers Studied by EPR
}

\author{
M. KEMPIŃSKI ${ }^{a}$, W. KEMPIŃSKI ${ }^{b}$ \\ AND M. ŚLIWIŃSKA-BARTKOWIAK ${ }^{a}$ \\ ${ }^{a}$ Institute of Physics, Adam Mickiewicz University \\ Umultowska 85, 61-614 Poznań, Poland \\ ${ }^{b}$ Institute of Molecular Physics, Polish Academy of Sciences \\ Smoluchowskiego 17, 60-179 Poznań, Poland
}

\begin{abstract}
We report results of EPR measurements of activated carbon fibers. Experiments made for pristine activated carbon fibers and activated carbon fibers with adsorbed molecules $\left(\mathrm{CCl}_{4}, \mathrm{C}_{6} \mathrm{H}_{5} \mathrm{NO}_{2}\right.$, and $\left.\mathrm{H}_{2} \mathrm{O}\right)$ confirmed the localized character of paramagnetic centers observed in the system. Pristine activated carbon fibers are characterized by single Lorentzian line. Broader component of EPR signal appears when guest molecules are adsorbed in nanopores. The strongest localization is observed for water-filled activated carbon fibers nanopores (with hydrophobic pore walls) where changes in distance between nanographite particles were monitored by the $g$-shift to higher values. This process is related to stronger spin-orbit interaction of electrons trapped at nanographite particles compressed by guest molecules.
\end{abstract}

PACS numbers: 42.81.Uv, 73.20.-r, 73.22.-f, 76.30.-v, 74.78.Na

\section{Introduction}

Activated carbon fibers (ACFs) possess nanocrystallites made up of graphene plates that create conducting particles nonuniformly distributed in space [1]. These particles, linked structurally but not necessarily electrically, create nanographitic porous system. For such system a model for granular metal is proposed with the tunneling conduction mechanism [2]. This mechanism leads to stronger localization of charge carriers at low temperature region. Different liquids $-\mathrm{CCl}_{4}$, 
$\mathrm{C}_{6} \mathrm{H}_{5} \mathrm{NO}_{2}$, and $\mathrm{H}_{2} \mathrm{O}$ - were chosen to find mutual host-guest interaction. For self-organized systems confined in nanometer scale, nonlinear dielectric effect measurements for $\mathrm{CCl}_{4}$ in $\mathrm{ACF}$ show divergence at transition, confirming the hexatic phase [3]. $\mathrm{C}_{6} \mathrm{H}_{5} \mathrm{NO}_{2}$ molecules were chosen because of possible indication of the charge transfer from nanographite particles to guest molecules by electron hyperfine interaction with $\mathrm{N}$ or $\mathrm{C}$ nuclei [4]. Contact of water molecules with hydrophobic surfaces of nanographite particles of ACFs is characterized by physisorption process which can modify the host system [5]. In contradiction to chemisorption, adsorption-desorption mechanism is easily implemented in the system of $\mathrm{H}_{2} \mathrm{O}$ ACFs by simple heating-pumping procedure. Water confined in nanometer porous system of ACFs can also help to solve the problem of possible contrast behavior of bulk and nanoscale matter. Transition from continuum to molecular behavior of water confined in nanometer channels of closed multiwall carbon nanotubes appears between 5 and $10 \mathrm{~nm}$ diameter of carbon tube [6].

\section{Experimental}

EPR measurements of ACFs were made using Radiopan ES/X spectrometer equipped with Oxford Instruments gas flow helium cryostat within the temperatures between $4.2 \div 300 \mathrm{~K}$. Microwave frequency was measured by microwave frequency counter with an accuracy of $5 \mathrm{kHz}$. The magnetic field was calibrated by tracking NMR magnetometer with an accuracy of $0.005 \mathrm{mT}$. ACFs samples were acquired from Osaka Gas Chemicals Co. Ltd, Japan.

In order to empty the porous system before guest molecules adsorption procedure, ACFs were evacuated by heating at $200^{\circ} \mathrm{C}$ and pumping with turbomolecular pump at $10^{-4}$ mbar.

\section{Results and discussion}

EPR measurements confirm localized character of paramagnetic centers observed in ACFs. Pristine ACFs are characterized by single Lorentzian line recorded below $100 \mathrm{~K}$ at $g=2.0031$ - value characteristic of carbon materials: graphite $g_{\perp}=2.0031$ [7], nanodiamond $g=2.0029$ [8], and $\mathrm{C}_{60}$ fullerene $g=2.0026$ [9]. When guest molecules are adsorbed in ACFs' voids, EPR spectrum becomes modified - a broader component of EPR signal appears for all studied systems - EPR signal of filled ACFs consists of three lines. Figure 1 shows characteristic EPR spectrum observed for liquid-filled $\mathrm{ACFs}$ at $20 \mathrm{~K}$. The strongest effect is observed for dipolar guest molecules: $\mathrm{C}_{6} \mathrm{H}_{5} \mathrm{NO}_{2}$ and $\mathrm{H}_{2} \mathrm{O}$. There was no EPR signal from guest molecules - no hyperfine splitting arising from interaction with nuclear spins of $\mathrm{N}$ or $\mathrm{H}$ was observed. No charge transfer from ACFs to guest molecules means that EPR modification concerns only nanographite particles. The narrow line (1) characterizes the pristine ACFs. Its line width and $g$-factor are temperature independent. Line (2) originates from nanographite particles (host) surrounded by 
molecules captured in nanopores (guest). Similarly to component (1), line width and $g$-factor are temperature independent [10]. Broadening of the line (2), compared to (1), is caused by the shorter relaxation time of the more dense system. Observed changes of line width, together with a small $g$-shift from $g_{\mathrm{e}}-$ spectroscopic splitting factor of free electron $\left(g-g_{\mathrm{e}}=\Delta g=6 \times 10^{-4}\right.$; mean value) for line (2) of $\mathrm{C}_{6} \mathrm{H}_{5} \mathrm{NO}_{2}$-filled ACFs, enabled us to estimate the size of graphite nanoparticles (approximately $1.34 \mathrm{~nm}$ ) in which distance between graphene plates could be modified by guests molecules [11]. Such an approach was also proposed for ultra dispersed diamond (UDD) [8] and fullerenes [12].

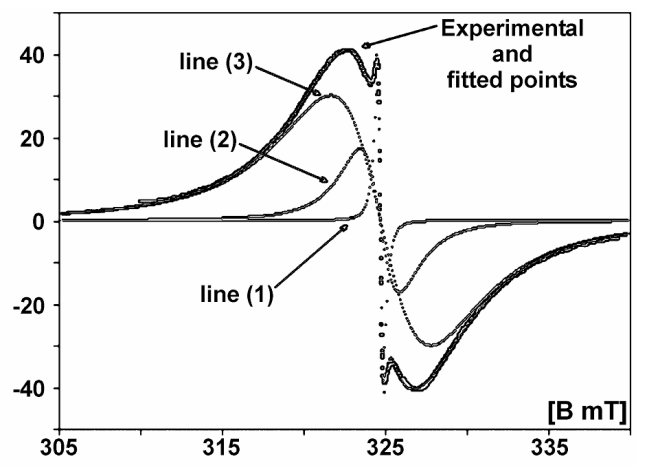

Fig. 1. EPR spectrum of ACF filled with nitrobenzene recorded at $20 \mathrm{~K}$ and frequency $9.102400 \mathrm{GHz}$ - experimental curve with the fit.

Line width and $g$-factor of the component (3) of observed EPR spectrum strongly depend on temperature - it can be explained as a surface effect of ACFs. No Dysonian shape of EPR line is observed for each component. It means that ACFs crystallites' size is lower than $3.2 \mu \mathrm{m}$ - penetration depth of microwave field [13]. Temperature dependences of integral intensities of each of the three lines are very close to the Curie law. Better fitting appears after taking into account additional number of spins from granular metals model $[2,14]$.

Absence of hyperfine splitting from interaction with $\mathrm{H}$ or $\mathrm{N}$ nuclei of guests molecules together with the Curie law for all three components confirm the strong localization of paramagnetic centers within ACFs' nanocrystallites at low temperature region. At this region $g$-factors of all three lines reach similar values for $\mathrm{CCl}_{4}$ - and $\mathrm{C}_{6} \mathrm{H}_{5} \mathrm{NO}_{2}$-filled ACFs (Fig. 2a). It confirms the low temperature stiffening of the whole system with small modification of nanographite particles' size [11]. Stronger modification appears when ACFs' porous system is filled with water molecules. It is shown in Fig. 2 b, where $g$-factors of lines (2) and (3) are shifted to higher values (for line (2) mean value of $g$-shift is $\Delta g=31 \times 10^{-4}$ ) when compared to line (1) as well as to lines (1), (2) and (3) from Fig. 2a (for ACFs + $\mathrm{C}_{6} \mathrm{H}_{5} \mathrm{NO}_{2}$ ). 

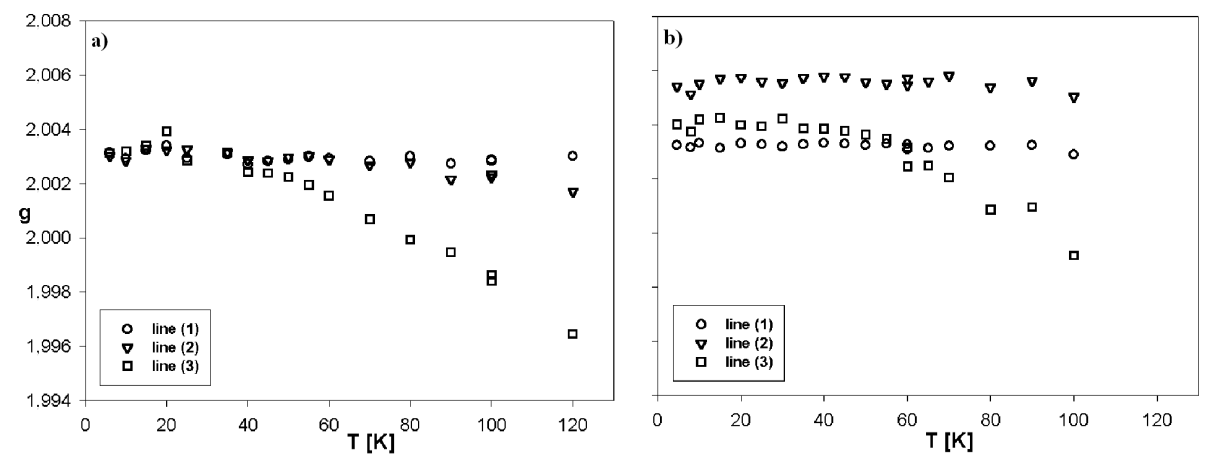

Fig. 2. (a) $g$-factor vs. temperature for $\mathrm{ACF}+\mathrm{C}_{6} \mathrm{H}_{5} \mathrm{NO}_{2}$ with mean value of $g$-shift $\Delta g=6 \times 10^{-4}$ for line (2). (b) $g$-factor vs. temperature for $\mathrm{ACF}+\mathrm{H}_{2} \mathrm{O}$ with mean value of $g$-shift $\Delta g=31 \times 10^{-4}$ for line (2).

Anisotropy of $g$-factor of bulk graphite, with strong difference between $g_{\perp}$ and $g_{\|}$, is a consequence of spin-orbit coupling [7, 13]. Spin-orbit interaction can also affect $g$-value when inter-graphene distances in 3D stacking order of basal planes are changed. When hydrophobic nanographite particles are surrounded by water molecules, suppression of paramagnetism of ACFs was observed — low spin state is caused by internal pressure of adsorbed water molecules [5].

In our experiments reduction of distances between graphene planes are detected by $g$-shift to higher values caused by stronger spin-orbit interaction which appears in compressed system of stacking graphene planes.

\section{Conclusions}

No hyperfine splitting from interaction with $\mathrm{H}$ and $\mathrm{N}$ nuclei together with almost perfect Curie law for all three components confirm the localization of paramagnetic centers within nanocrystallites of ACFs structure. No charge transfer from host to guest means that only weak van der Waals interactions appear in the system of ACFs with $\mathrm{C}_{6} \mathrm{H}_{5} \mathrm{NO}_{2}$ and $\mathrm{CCl}_{4}$ adsorbed. For ACFs $+\mathrm{H}_{2} \mathrm{O}$ nanocrystallites' shrinkage effect arising from hydrophobic properties of graphite was observed by examining the $g$-value shift.

\section{References}

[1] A.M. Rao, A.W.P. Fung, M.S. Dresselhaus, M. Endo, J. Mater. Res. 7, 1788 (1992).

[2] A.W.P. Fung, A.M. Rao, K. Kuriyama, M.S. Dresselhaus, G. Dresselhaus, M. Endo, N. Shindo, J. Mater. Res. 8, 489 (1993).

[3] R. Radhakrishnan, K.E. Gubbins, M. Śliwińska-Bartkowiak, Phys. Rev. Lett. 89, 076101 (2002). 
[4] T.L. Chu, G.E. Pake, D.E. Paul, J. Townsend, S.I. Weissman, J. Phys. Chem. 57, 504 (1953).

[5] H. Sato, N. Kawatsu, T. Enoki, M. Endo, R. Kobori, S. Maruyama, K. Kaneko, Solid State Commun. 125, 641 (2003).

[6] N. Naguib, H. Ye, Y. Gogotsi, A.G. Yazicioglu, C.M. Megaridis, M. Yoshimura, Nano Lett. 4, 2237 (2004).

[7] J. Stankowski, L. Piekara-Sady, W. Kempiński, O. Huminiecki, P.B. Szczaniecki, Full. Sci. Technol. 5, 1203 (1997).

[8] A.I. Shames, A.M. Panich, W. Kempiński, A.E. Alexenskii, M.V. Baidakova, A.T. Dideikin, V.Y. Osipov, V.I. Siklitski, E. Osawa, M. Ozawa, J. Phys. Chem. Solids 63, 1993 (2002).

[9] W. Kempiński, P. Scharff, J. Stankowski, L. Piekara-Sady, Z. Trybuła, Physica C 274, 232 (1997).

[10] M. Kempiński, M. Śliwińska-Bartkowiak, W. Kempiński, Mol. Phys. Rep. 37, 136 (2003).

[11] M. Kempiński, M. Śliwińska-Bartkowiak, W. Kempiński, in: Nonlinear Dielectric Phenomena in Complex Liquids, Ed. S.J. Rzoska, V.P. Zhelezny, Kluwer Academic Publishers, Netherlands 2004, p. 387.

[12] J. Stankowski, L. Piekara-Sady, W. Kempiński, Appl. Magn. Res. 19, 539 (2000).

[13] G. Wagoner, Phys. Rev. 118, 647 (1960).

[14] M. Kempiński, submitted to Solid State Commun. 